\title{
Enhanced brain targeting of temozolomide in polysorbate-80 coated polybutylcyanoacrylate nanoparticles
}

\author{
Xin-Hua Tian' \\ Xiao-Ning Lin' \\ Feng Wei ${ }^{1}$ \\ Wei Feng' \\ Zhi-Chun Huang' \\ Peng Wang' \\ Lei Ren ${ }^{2}$ \\ Yi Diao' \\ 'Department of Neurosurgery, \\ Zhongshan Hospital of Xiamen \\ University, Xiamen, People's \\ Republic of China; ${ }^{2}$ Research Center \\ of Biomedical Engineering, Xiamen, \\ People's Republic of China
}

This article was published in the following Dove Press journal:

International Journal of Nanomedicine

22 February 201I

Number of times this article has been viewed

\begin{abstract}
Background: Polybutylcyanoacrylate (PBCA) nanoparticles coated with polysorbate- 80 have been extensively proposed for delivering drugs into the animal brain and have shown great potential for therapeutic applications. In this study, we made an attempt to deliver the chemotherapeutic drug, temozolomide, into the brain by using PBCA nanoparticles. The physicochemical characteristics, in vitro release, and brain targeting ability of the drug-loaded nanoparticles were investigated.
\end{abstract}

Results: Our results show that a significantly higher concentration of temozolomide in the form of polysorbate-80-coated PBCA nanoparticles was observed in the brain $(P<0.05)$ in comparison with the free drug.

Conclusion: This study indicates that polysorbate- 80 coated PBCA nanoparticles could be a feasible carrier for temozolomide delivery to the brain. It is anticipated that the developed formulation may improve on targeted therapy for malignant brain tumors in the future.

Keywords: temozolomide, polybutylcyanoacrylate, nanoparticles, polysorbate-80, brain targeting

\section{Introduction}

The treatment of malignant brain tumors is one of the most challenging issues in central nervous system diseases, especially glioblastoma, for which the median survival time of patients is still less than 1 year at present. ${ }^{1}$ The failure of chemotherapy in these tumors is attributed not to the chemotherapeutic activity of the available drugs, but to the existence of an insurmountable obstacle, ie, the blood-brain barrier. The bloodbrain barrier, formed by the endothelial cells of the brain capillaries coupled together by tight junctions (zonulae occludentes), inhibits the transport of $98 \%$ of all smallmolecule drugs and $100 \%$ of large-molecule pharmaceutics into the brain. ${ }^{2,3}$ Therefore, it is difficult to deliver effective drug concentrations to the tumor site in the brain.

In the past few years, a number of different approaches have been developed for drugs to overcome this barrier. The methods include direct injection into the brain, ${ }^{4}$ osmotic opening of the tight junctions, ${ }^{5}$ drug structural modifications, ${ }^{6}$ chemical drug delivery systems, and nanoparticulate carriers, such as liposomes, ${ }^{7,8}$ solid lipid nanoparticles, or polymeric nanoparticles. ${ }^{9,10}$ Among these various noninvasive strategies, polymeric nanoparticles seem to be one of the most promising and exciting techniques for central nervous system drug delivery. Polymers are made of natural or synthetic materials, with sizes ranging from 1 to $1000 \mathrm{~nm}$, into which the drug load is encapsulated, passively adsorbed, or chemically linked to the surface. ${ }^{10,11}$ On the one hand, nanoparticles can protect the entrapped drugs from degradation so as to increase the 
drug concentration at the targeted site, and on the other hand, by modifying the property of the nanoparticle surfaces with surfactants, nanoparticles can improve drug delivery by avoiding uptake by the reticuloendothelial system in the body. ${ }^{12}$ Therefore, nanoparticles are increasingly demonstrating their advantage of effective transporting of various different drugs across the blood-brain barrier on the basis of their small size and appropriate surface functionalization. ${ }^{11}$

Polybutylcyanoacrylate (PBCA), a minimally toxic, biocompatible, and biodegradable polymer, has been extensively investigated as a drug carrier in recent years. It is easy to synthesize and scale up, and possesses the abilities of altering the biodistribution of loaded drugs and modulating drug release characteristics. ${ }^{13,14}$ Most importantly, many studies have showed that PBCA nanoparticles are able to transport therapeutic agents in significant quantities across the blood-brain barrier into the brain when coated with surfactant polysorbate- 80 on their surfaces. These drugs include anticancer agents, ${ }^{13,15}$ analgesics, ${ }^{16}$ peptides,${ }^{17} \mathrm{~N}$-methylD-aspartate receptor antagonists, ${ }^{18}$ antibiotics, and nonsteroidal anti-inflammatory drugs. ${ }^{19,20}$ The precise mechanism of delivery of drug-loaded polysorbate- 80 coated PBCA nanoparticles is still not fully clarified. It appears to be achieved by covalent coupling with apolipoprotein E, A-I, or B-100, followed by receptor-mediated endocytosis of the nanoparticles by the brain capillary endothelial cells. ${ }^{21,22}$

Temozolomide (3,4-dihydro-3-methyl-4-oxoimidazo [5,1-d]-as-tetrazine-8-carboxamide [TMZ]; see Figure 1), first synthesized by the UK Cancer Research Campaign in the 1980s, is a cytotoxic alkylating agent with a chemical formula of $\mathrm{C}_{6} \mathrm{H}_{6} \mathrm{~N}_{6} \mathrm{O}_{2}$ and a molecular weight of 194.15. ${ }^{23}$ The solubility of TMZ in normal saline is $3.1 \mathrm{mg} / \mathrm{mL} .{ }^{24}$ It has been shown to be one of the most effective antineoplastic

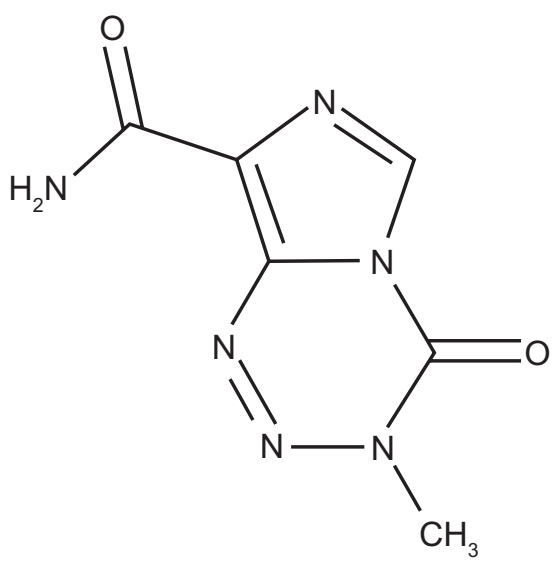

Figure I Chemical structure of TMZ. agents for treating high-grade glioma and metastatic melanoma. Unfortunately, due to its short half-life of approximately 1.8 hours in plasma, TMZ has to be administered with high systemic doses to reach therapeutic brain levels, which simultaneously brings about a series of side effects, including bone marrow depression, oral ulcerations, nausea, vomiting, fatigue, and headache. ${ }^{25}$ In order to get better efficacy for treating brain tumors and reduce related toxicity and side effects during chemotherapy, it is necessary to identify the most efficient way to achieve drug targeting for meeting the requirements of modern therapy, and perhaps polymeric nanoparticles as a drug delivery system is a good choice. Hence, in the present research work, the possibility of targeting delivery of TMZ with polysorbate- 80 coated PBCA nanoparticles into the brain was investigated.

\section{Material and methods Materials}

TMZ powder was a gift from Jiangsu Tasly Diyi Pharmaceutical Co Ltd (Huaian, China). The monomer n-butylcyanoacrylate (n-BCA) was purchased from Guangzhou Baiyun Medical Adhesive Co Ltd (Guangzhou, China). Polysorbate-80 was obtained from Sigma Chemical Co (St. Louis, MO). Pluronic F68 and D-mannitol were purchased from Bio Basic Inc (Markham, Canada). All other materials and reagents used were of analytical/high-pressure liquid chromatography grade and without further purification. The glassware were thoroughly cleaned and rinsed with deionized water.

\section{Preparation of nanoparticles}

The preparation of PBCA nanoparticles containing TMZ was performed according to the method of emulsion polymerization, ${ }^{17}$ with minor modifications. Briefly, under constant magnetic stirring, 1\% n-BCA monomer was added dropwise to $10 \mathrm{~mL}$ of solution ( $\mathrm{pH} 2.5$ adjusted with $0.1 \mathrm{~N} \mathrm{HCl}$ ) containing 1\% Pluronic F68 and $5 \mathrm{mg}$ TMZ. After stirring at $500 \mathrm{rpm}$ for 4 hours at room temperature, the $\mathrm{pH}$ value of the nanoparticle suspension was adjusted to $5.0 \pm 0.5$ with $0.1 \mathrm{~N}$ sodium hydroxide solution, and then the stirring was continued for another 30 minutes to complete the reaction. The resulting suspension was filtered through a sintered glass filter (pore size $10 \mu \mathrm{m}$ ) to remove any agglomerates. Subsequently, the colloidal suspension was frozen by dry ice and freeze-dried (FDU-1200; EYELA, Tokyo, Japan) after addition of $3 \%$ mannitol as a cryoprotector. The lyophilized powder was resuspended in saline containing $1 \%$ anhydrous glucose. For surfactant coating, 1\% polysorbate-80 was 
added and the mixture was incubated for 30 minutes at ambient temperature under constant stirring prior to administration. The drug-free nanoparticles were prepared using the same technique. ${ }^{16}$

\section{Preparation of solution of free TMZ}

TMZ solution was prepared by dissolving $5 \mathrm{mg}$ of TMZ with dimethyl sulfoxide in $0.9 \%$ saline $(20 \%, \mathrm{v} / \mathrm{v})$ for intravenous administration and administered within four hours. ${ }^{26}$

\section{Analysis of particle size and surface charge}

The particle sizes and surface charges (zeta potentials) of the nanoparticles were measured on a Nano-ZS Zetasizer dynamic light scattering detector (Malvern Instruments, Malvern, UK) equipped with a $4.0 \mathrm{~mW}$ internal laser. All measurements were performed at $25^{\circ} \mathrm{C}$ at a scattering angle of $173^{\circ}$. Prior to measurement, the suspensions were diluted with deionized water to an appropriate concentration for better measurement. Values of the particle sizes and zeta potentials are presented as mean \pm standard deviation (SD) from three replicate samples. The width of the size distributions were characterized by the polydispersity index.

\section{Particulate morphology}

Nanoparticle suspension was dropped onto a copper grid and dyed with $2 \%$ phosphotungstic acid, and the morphology of the nanoparticles was then observed by negative staining under a transmission electron microscope (JEM-2100HC; JEOL, Tokyo, Japan).

\section{Fourier transform infrared spectroscopy}

The Fourier transform infrared spectra were recorded using a AVATAR360 FTIR spectrometer (Nicolet, Madison, WI) at a resolution of $4 \mathrm{~cm}^{-1}$, and 256 scans were taken in the range 400-4000 $\mathrm{cm}^{-1}$. The sample of the nanoparticles (with and without drug) or TMZ used for the Fourier transform infrared spectroscopic characterization were prepared by grinding the dry specimens with spectroscopic grade $\mathrm{KBr}$ and pressing them to form disks.

\section{Determination of drug loading and entrapment efficiency}

Before freeze-drying, the nanoparticle suspension was centrifuged (Avanti J-E; Beckman Coulter, Brea, CA) at 20,000 rpm for 30 minutes at $4{ }^{\circ} \mathrm{C}$. The concentrations of TMZ in the supernatant were diluted with $0.1 \mathrm{~N} \mathrm{HCl}$ and then measured at a wavelength of $329 \mathrm{~nm}$ using an ultraviolet spectrophotometer (Beckman Coulter). The entrapment efficiency was calculated as the difference between the amount of drug entrapped in the nanoparticles and the total amount of drug, while drug loading was determined as drug analyzed in the nanoparticles versus the weight of the final polymer mass after drying.

\section{Evaluation of in vitro release}

The in vitro release was studied using a dialysis bag diffusion technique. ${ }^{27,28}$ The TMZ-loaded nanoparticle suspension (coated and uncoated with polysorbate-80) or TMZ solution was placed in cellulose dialysis bags (cutoff $3.5 \mathrm{kDa}$; Solarbio), which were sealed at both ends, and then the bags were dipped into the crystallizing dishes containing $200 \mathrm{~mL}$ phosphate-buffered saline ( $\mathrm{pH}$ 6.8), which were maintained at $37^{\circ} \mathrm{C}$ and constantly stirred at $100 \mathrm{rpm}$. The crystallizing dishes were closed to prevent evaporation of the release medium. At regular time intervals, $2 \mathrm{~mL}$ of dissolution medium was withdrawn and the same volume of fresh phosphatebuffered saline was added accordingly. The amount of TMZ in the medium was measured by high-pressure liquid chromatography analysis.

\section{Animal testing}

Healthy adult Wistar rats weighing 180-220 g were purchased from the Medical School Laboratory Animal Center at Xiamen University and used for body distribution studies. They had access to food and water ad libitum. All animals were provided with humane care according to the Principles of Laboratory Animal Care formulated by the National Society for Medical Research and the Guide for the Care and Use of Laboratory Animals prepared by the Institute of Laboratory Animal Resources and published by the National Institutes of Health (Publication 86-32, revised 1985). The rats were randomly divided into four groups with six animals per group. Group 1 was injected with TMZ solution into the tail vein, Group 2 was treated with TMZ-PBCA nanoparticles, Group 3 was given 1\% polysorbate- 80 coated TMZ-PBCA nanoparticles, and Group 4 served as the control. All formulations were administered at a dose of $10 \mathrm{mg}$ of TMZ/kg. ${ }^{27}$ One hour after injection, the rats were anesthesitized with ethyl ether and sacrificed by decapitation..$^{29,30}$ The selected organs, ie, the brain, lungs, heart, liver, spleen, and kidneys, were quickly dissected and stored at $-20^{\circ} \mathrm{C}$. The amount of drug in each organ was measured by high-pressure liquid chromatography analysis. 


\section{High-pressure liquid chromatography analysis of TMZ}

TMZ content was measured by high-pressure liquid chromatography/ultraviolet absorption (Waters 2695 and 2996; Waters, Milford, MA) at a wavelength of $329 \mathrm{~nm}$, and a Hypersil C18 ODS column ( $5 \mu \mathrm{m}, 250 \mathrm{~mm} \times 4.6 \mathrm{~mm}$; Elite, Dalian, China) was used. The mobile phase methanol $/ 0.5 \%$ glacial acetic acid solution (10:90, v/v) was delivered at a flow rate of $1 \mathrm{~mL} / \mathrm{min}$ at ambient temperature. ${ }^{27}$ All organ samples were homogenized, and each homogenate sample was then mixed with acetone and vortexed for two minutes. After centrifugation at $4000 \mathrm{rpm}$ for 15 minutes at $4^{\circ} \mathrm{C}$, the supernatant was evaporated to dryness under nitrogen stream at $60^{\circ} \mathrm{C}$. Finally, the residue was dissolved in $200 \mu \mathrm{L}$ mobile phase and $20 \mu \mathrm{L}$ of the solution was injected into the highpressure liquid chromatography system for analysis.

\section{Statistical analysis}

The results were presented as mean \pm SD calculated over at least three data points. Statistical significance of differences was processed by one-way analysis of variance and a subsequent post hoc Tukey comparison. A value of $P<0.05$ was considered to be statistically significant.

\section{Results}

\section{Physicochemical characteristics of nanoparticles}

Figure 2 shows the transmission electron microscopy morphology of TMZ-PBCA nanoparticles with an average diameter of about $120 \mathrm{~nm}$. Most nanoparticles could be observed to be of spherical or ellipsoidal shape with a relatively smooth surface. Coinciding with transmission electron microscopic photography, dynamic light scattering results indicated that the average size of the TMZ-PBCA nanoparticles was $135.8 \pm 11.3 \mathrm{~nm}(\mathrm{n}=9)$, and polydispersity indices
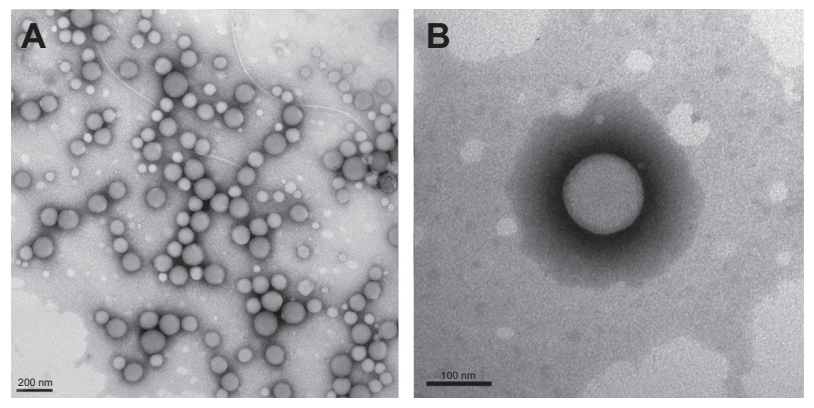

Figure 2 TEM image of TMZ-PBCA-NPs prepared by emulsion polymerization. Bars represent $200 \mathrm{~nm} \mathrm{~A}$ ) and $100 \mathrm{~nm} \mathrm{~B}$ ). ranged from 0.191 to 0.306 . The surface of nanoparticles carried negative charges, with a mean zeta potential of $-24.8 \pm 2.2 \mathrm{mV}(\mathrm{n}=9)$ in deionized water. The entrapment efficiency was $44.23 \pm 2.04 \%(n=3)$, and drug loading was $2.80 \pm 0.05 \%(\mathrm{n}=3)$.

Figure 3 shows the Fourier transform infrared spectra of TMZ, PBCA nanoparticles, and TMZ-PBCA nanoparticles obtained in the present study and it was performed to verify drug entrapped into the nanoparticles. As revealed in spectrum, the main four functional groups of PBCA nanoparticles were $\mathrm{C}-\mathrm{H}, 2950 \mathrm{~cm}^{-1}, \mathrm{C} \equiv \mathrm{N}, 2250 \mathrm{~cm}^{-1}, \mathrm{C}=\mathrm{O}$, $1750 \mathrm{~cm}^{-1}$, and $\mathrm{C}-\mathrm{O}, 1250 \mathrm{~cm}^{-1}$. In the Fourier transform infrared spectra of TMZ, two strong characteristic bands were observed at $3400 \mathrm{~cm}^{-1}$ and $1610 \mathrm{~cm}^{-1}$. The first was due to the N-H stretching mode and the second corresponded to the $\mathrm{C}=\mathrm{C}$ or $\mathrm{C}=\mathrm{N}$ stretching vibration, while in the spectrum of TMZ-PBCA nanoparticles, the presence of the characteristic bands of TMZ and PBCA nanoparticles was found at the same place simultaneously.

\section{Evaluation of in vitro release}

The release profiles of TMZ solution, TMZ-PBCA, and TMZ-PBCA nanoparticles coated with polysorbate- 80 are shown in Figure 4. As shown in the releasing curve, we observed that approximately $80 \%$ of TMZ had been released from TMZ solution after one hour, but the cumulative release of the drug from nanoparticles was slow. By 24 hours, the total cumulative releases from the TMZ-PBCA nanoparticles and TMZ-PBCA nanoparticles coated with polysorbate- 80 were $66.03 \pm 1.61 \%$ and $62.97 \pm 1.40 \%$, respectively.

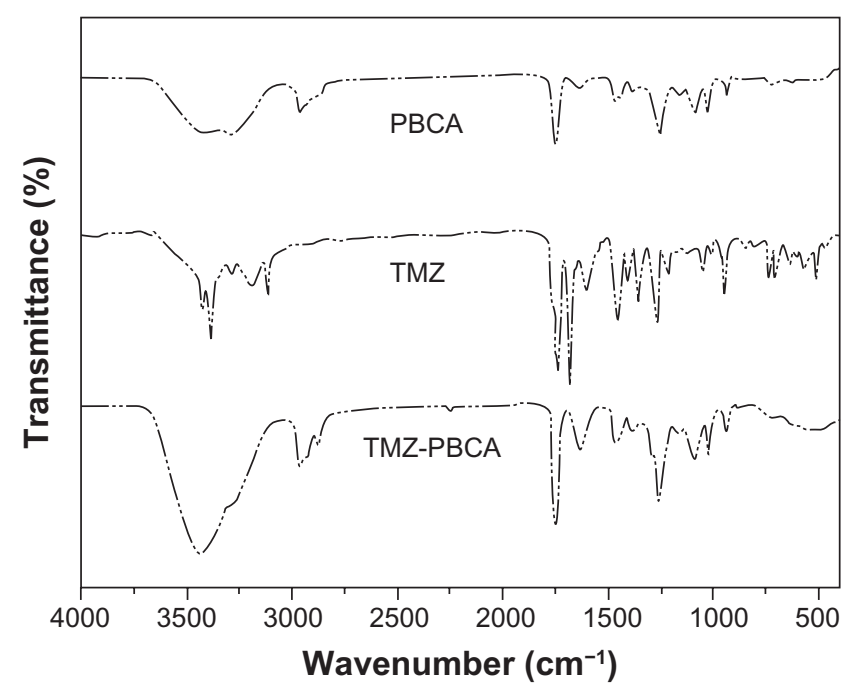

Figure 3 FTIR spectra of TMZ, PBCA-NPs and TMZ-PBCA-NPs. 


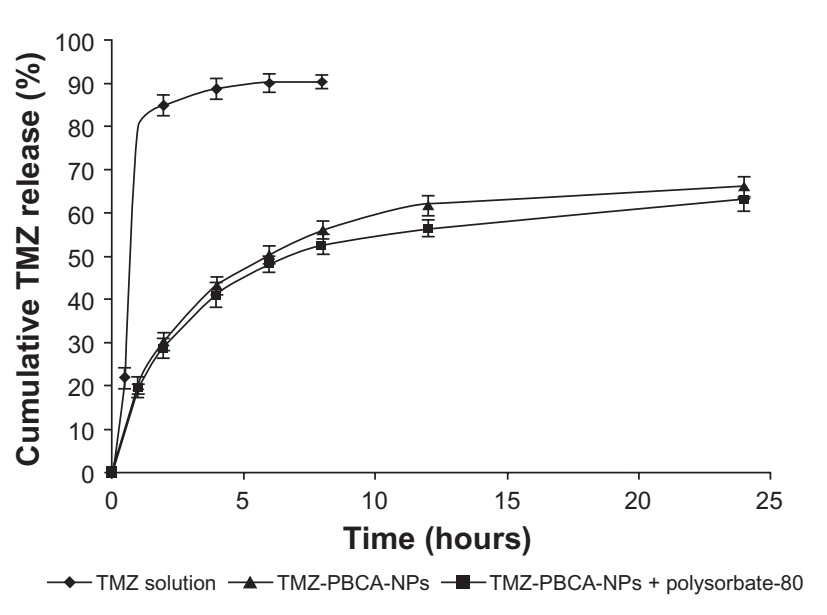

Figure 4 In vitro release profile of TMZ solution, TMZ-PBCA-NPs and TMZ-PBCANPs + polysorbate- 80 .

Table 1 shows the different fitting model results of the nanoparticles without coating and coated with polysorbate-80. Both were fitted to Higuchi and Ritger-Peppas equations better with higher $\mathrm{r}^{2}$ values.

\section{Animal testing}

Table 2 and Figure 5 show the TMZ concentrations $(\mu \mathrm{g} / \mathrm{g})$ in different organs after intravenous administration in rats. When the rats were given TMZ solution, the concentrations of TMZ in the heart, liver, spleen, lungs, and kidneys after one hour were $3.29 \pm 0.23,3.26 \pm 0.35,2.11 \pm 0.17,1.95 \pm 0.12$, and $5.17 \pm 0.47 \mu \mathrm{g} / \mathrm{g}$, respectively. However, lower concentrations were observed in the heart and kidneys $(2.19 \pm 0.29$, $1.68 \pm 0.26 \mu \mathrm{g} / \mathrm{g}$ and $4.09 \pm 0.37,3.71 \pm 0.30 \mu \mathrm{g} / \mathrm{g}$ ) after treating the rats with TMZ-PBCA nanoparticles and nanoparticles coated with polysorbate-80, which is in accordance with data already reported. ${ }^{13}$ Higher concentrations observed in the liver, spleen, and lungs when TMZ was bound with nanoparticles were caused by the reticuloendothelial system in these organs. In the brain, compared with TMZ solution, both types of nanoparticles, especially nanoparticles coated with polysorbate-80, significantly increased the accumulation of the drug by 2.29 -fold $(1.10 \pm 0.19 \mu \mathrm{g} / \mathrm{g}$ versus $0.48 \pm 0.11 \mu \mathrm{g} / \mathrm{g})$.

Table I The different release model of TMZ-PBCA nanoparticles (I) and TMZ-PBCA nanoparticles + polysorbate-80 II $(n=3)$

\begin{tabular}{llllll}
\hline Formulation & \multicolumn{2}{l}{ Model } & & & \\
\cline { 2 - 6 } & $\begin{array}{l}\text { First } \\
\text { order }\end{array}$ & $\begin{array}{l}\text { Hixson- } \\
\text { Crowell }\end{array}$ & Higuchi & Ritger- Peppas \\
\cline { 2 - 6 } & $\mathbf{r}$ & $\mathbf{r}$ & $\mathbf{r}$ & $\mathbf{r}$ & $\mathbf{n}$ \\
\hline $\mathrm{I}$ & 0.865 & 0.843 & 0.945 & 0.967 & 0.383 \\
$\mathrm{II}$ & 0.868 & 0.845 & 0.947 & 0.966 & 0.382 \\
\hline
\end{tabular}

\section{Discussion}

PBCA nanoparticles containing TMZ were prepared by emulsion polymerization and presented a relatively homogeneous shape (Figure 2), with a mean size lower than $200 \mathrm{~nm}$. These results are in agreement with recently reported data. ${ }^{28,31,32}$ Pluronic F68, which was used as a steric stabilizer for obtaining a stable nanoparticle form, would increase the size of the nanoparticles when its percentage was more than $3 \% .{ }^{31}$ Additionally, the $\mathrm{pH}$ value would be another factor affecting the particle size. Both below pH 2.0 and above pH 3.0 would increase the particle size significantly. ${ }^{33,34}$ Therefore, we chose Pluronic F68 1\% and pH 2.5 as experimental conditions in the study. The zeta potential, which reflects particle stability, was negative $(-24.8 \pm 2.2 \mathrm{mV})$, indicating sufficient repulsion between particles to avoid their aggregation and formation of a stable nanoparticle suspension. ${ }^{35}$ The entrapment efficiency of the prepared nanoparticles in our study was not very high, and about $44.23 \pm 2.04 \%$ of the $\mathrm{TMZ}$ in the preparation was bound to the nanoparticles. This result might be due to the amphiphilic nature of TMZ, which is relatively highly lipophilic and somewhat soluble in water. ${ }^{23}$ Another reason may be that the TMZ bound to the nanoparticles mainly depended on physical adsorption and entrapment rather than covalent binding, as the results of the spectrum (Figure 3) suggest. Other preparation methods, like interfacial polymerization or miniemulsion polymerization, might be helpful to achieve a higher entrapment efficiency, ${ }^{15,16}$ but further studies still need to be done to investigate this further.

The release pattern of TMZ-PBCA nanoparticles without coating and coated with polysorbate- 80 was noted to be typically biphasic, while the TMZ solution was a quick releasing process. The initial burst in the first hour indicates that some of the drug molecules could be on the surface of the nanoparticles and the following sustained release might be due to the drug entrapped in the nanoparticles. The mechanism of drug release from PBCA nanoparticles would be Fickian diffusion according to the $n$ values $(n<0.45)$ calculated by the Ritger-Peppas model, as shown in Table 1. This result is in accordance with earlier findings. ${ }^{14,33}$ In addition, it is obvious from the Figure 4 that coating with $1 \%$ polysorbate- 80 would slightly decrease drug release from the nanoparticles. This might be caused by leakage of drug into the medium during the process of coating. ${ }^{28}$ Furthermore, the reason for the low total cumulative release of the two kinds of nanoparticles might be the low amount of drug in the particles discussed here. 
Table 2 Equations of standard curve of the organs and body distribution (in $\mu g$ drug per $g$ tissue, $\mu g / g$ ) after intravenous administration of different temozolomide formulations in rats $(n=6)$

\begin{tabular}{|c|c|c|c|c|}
\hline \multirow[t]{2}{*}{ Organ } & \multirow[t]{2}{*}{ Equation of standard curve } & \multicolumn{3}{|c|}{ Formulation } \\
\hline & & I & II & III \\
\hline Heart & $Y=2.10 \times 10^{5} X+1.51 \times 10^{4}(r=0.9974)$ & $3.29 \pm 0.23$ & $2.19 \pm 0.29$ & $1.68 \pm 0.26$ \\
\hline Liver & $Y=1.86 \times 10^{5} X+1.32 \times 10^{4}(r=0.9963)$ & $3.26 \pm 0.35$ & $4.41 \pm 0.46^{b}$ & $4.84 \pm 0.47^{b}$ \\
\hline Spleen & $Y=2.03 \times 10^{5} X+2.39 \times 10^{4}(r=0.9937)$ & $2.11 \pm 0.17$ & $3.22 \pm 0.3 \mathrm{I}^{\mathrm{b}}$ & $3.08 \pm 0.25^{b}$ \\
\hline Lungs & $Y=2.09 \times 10^{5} X+2.01 \times 10^{4}(r=0.9969)$ & $1.95 \pm 0.12^{\mathrm{a}}$ & $2.47 \pm 0.24^{b}$ & $2.20 \pm 0.32^{\mathrm{a}, \mathrm{b}}$ \\
\hline Kidneys & $Y=1.85 \times 10^{5} X+2.63 \times 10^{4}(r=0.9922)$ & $5.17 \pm 0.47$ & $4.09 \pm 0.37^{b}$ & $3.71 \pm 0.30^{\mathrm{b}}$ \\
\hline Brain & $Y=2.00 \times 10^{5} X+1.97 \times 10^{4}(r=0.9972)$ & $0.48 \pm 0.11$ & $0.71 \pm 0.12$ & $1.10 \pm 0.19$ \\
\hline
\end{tabular}

Notes: I = TMZ solution; II = TMZ-PBCA nanoparticles; I = TMZ-PBCA nanoparticles + polysorbate- 80 . Level of significance according to the Tukey test $P<0.05$. This level is reached between the different groups unless indicated by superscript letters: ${ }^{a}$ Nonsignificant difference between I and III; ${ }^{b}$ Nonsignificant difference between II and III.

According to the in vivo results of our experiments, TMZ-PBCA nanoparticles coated with polysorbate- 80 were able to promote better biodistribution of the drug into the brain, as compared with the free drug solution and uncoated drug-loaded nanoparticles. This result is in agreement with earlier data reported for other drugs. Lobenberg and Ambruosi reported that a higher brain concentration of azidothymidine and doxorubicin was observed one hour after administration of the coated nanoparticles in rats. ${ }^{29,30}$ It was also observed that methotrexate bound to polysorbate-80-coated nanoparticles could significantly increase drug levels in the brain. ${ }^{36}$ Other drugs that have been successfully transported across the blood-brain barrier and improve drug level in brain by using PBCA nanoparticles coated with polysorbate- 80 include dalargin, ${ }^{17}$ loperamide, ${ }^{16}$ tubocurarine, ${ }^{37} \mathrm{MRZ} 2 / 576$, and gemcitabine. ${ }^{18,32}$ According to a recent study, ${ }^{38}$ polysorbate- 80 played a specific role in brain targeting, and was essential for the delivery of drug-loaded nanoparticles into the brain.

The exact mechanism of nanoparticle-mediated transport of drugs across the blood-brain barrier has yet to be determined.

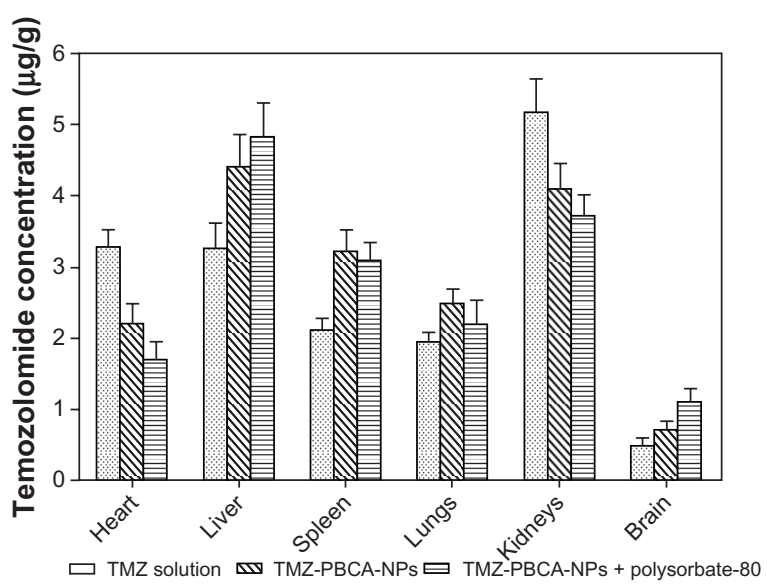

Figure 5 In vivo different tissue distribution of temozolomide concentrations $(\mu g / g)$ after intravenous injection of TMZ solution, TMZ-PBCA-NPs and TMZ-PBCANPs + polysorbate-80.
Several possibilities that could explain the mechanisms have been proposed. These include: an increased retention time of the nanoparticles in the brain capillaries which could enhance transport across the blood-brain barrier; the surfactant effect would lead to endothelial cell membrane fluidization and to enhanced drug permeability; the nanoparticles could induce opening of the tight junctions between the endothelial cells; the nanoparticles might be endocytosed by the endothelial cells to deliver the drug into the brain; the drug-loaded nanoparticles could be delivered into the brain by transcytosis; and the polysorbate- 80 could inhibit the P-glycoprotein efflux pump. ${ }^{39}$ Among these, the most likely mechanism is endocytosis, which has been supported by many studies in the past few years. ${ }^{21,22,39}$ Nanoparticles coated with polysorbate- 80 could covalently couple with apolipoprotein E, A-I, or B-100 in the blood stream and then mimic low-density lipoprotein particles to interact with the lowdensity lipoprotein receptor, leading to their uptake by brain capillary endothelial cells. Finally, the drug could be delivered by passive diffusion from endothelial cells to the brain and generate therapeutic effects.

In our experimental results, it was also observed that uncoated nanoparticles would lead to a significantly higher concentration of TMZ in the liver, spleen, and lungs after intravenous injection to rats in comparison with the free drug solution (Table 2). The reason is that these organs are rich in the phagocytic cells of the reticuloendothelial system, by which drug carriers with an average size below $7 \mu \mathrm{m}$ are easily taken up in blood stream. However, the drug nanoparticle concentrations were significantly lower in the heart and kidney than for the TMZ solution (Table 2), especially the nanoparticles coated with polysorbate- 80 . This indicates that TMZ-PBCA nanoparticles coated with polysorbate- 80 might decrease cardiac and renal accumulation of TMZ and reduce the toxicity of TMZ to the heart and kidney. This result is in accordance with previously reported data. ${ }^{13,27}$ 
In summary, the in vitro results presented here confirm the sustained release effect of TMZ-PBCA nanoparticles against TMZ solution. Moreover, our present study confirms earlier findings that only drug-loaded nanoparticles coated with polysorbate- 80 could generate a significant improvement of drug levels in the brain. Altogether, our results confirm our hypothesis that the brain-targeting ability of TMZ would be enhanced when bound to PBCA nanoparticles coated with polysorbate- 80 .

\section{Conclusion}

The present results demonstrate the feasibility of encapsulating the alkylating agent, TMZ, into PBCA nanoparticles by polymerization. Compared with TMZ solution, TMZ-PBCA nanoparticles exhibited sustained release in vitro. Furthermore, based on the pattern of distribution in body organs, higher concentrations of TMZ can be detected in the brain after binding to PBCA nanoparticles coated with polysorbate-80, which may be more useful for treating brain tumors. The prepared formulation may also reduce the toxicity of chemotherapy. However, to serve as a drug delivery system in the treatment of malignant brain tumors, further investigations are required.

\section{Acknowledgments}

This work was financially supported by the Natural Science Foundation of Fujian Province of China and the fund project of the Xiamen Science and Technology Bureau of China. The authors thank Xuesong Cao (Research Center of Anticancer, Xiamen University) for her helpful advice regarding preparation of the manuscript.

\section{Disclosure}

The authors report no conflicts of interest in this work.

\section{References}

1. Beduneau A, Saulnier P, Benoit JP. Active targeting of brain tumors using nanocarriers. Biomaterials. 2007;28(33):4947-4967.

2. Pardridge WM. Blood-brain barrier delivery. Drug Discov Today. 2007; 12(1-2):54-61.

3. Wolburg H, Lippoldt A. Tight junctions of the blood-brain barrier: Development, composition and regulation. Vascul Pharmacol. 2002; 38(6):323-337.

4. Fauchon F, Chiras J, Poisson M, et al. Intra-arterial chemotherapy by cisplatin and cytarabine after temporary disruption of the blood-brain barrier for the treatment of malignant gliomas in adults. J Neuroradiol. 1986;13(3):151-162.

5. Kroll RA, Neuwelt EA. Outwitting the blood-brain barrier for therapeutic purposes: Osmotic opening and other means. Neurosurgery. 1998;42(5):1083-1099.

6. Lambert DM. Rationale and applications of lipids as prodrug carriers. Eur J Pharm Sci. 2000;11 Suppl 2:S15-S27.

7. Bodor N, Buchwald P. Recent advances in the brain targeting of neuropharmaceuticals by chemical delivery systems. Adv Drug Deliv Rev. 1999;36(2-3):229-254.
8. Huwyler J, Wu D, Pardridge WM. Brain drug delivery of small molecules using immunoliposomes. Proc Natl Acad Sci U S A. 1996; 93(24):14164-14169.

9. Blasi P, Giovagnoli S, Schoubben A, Ricci M, Rossi C. Solid lipid nanoparticles for targeted brain drug delivery. Adv Drug Deliv Rev. 2007;59(6):454-477.

10. Tosi G, Costantino L, Ruozi B, Forni F, Vandelli MA. Polymeric nanoparticles for the drug delivery to the central nervous system. Expert Opin Drug Deliv. 2008;5(2):155-174.

11. Tiwari SB, Amiji MM. A review of nanocarrier-based CNS delivery systems. Curr Drug Deliv. 2006;3(2):219-232.

12. Gref R, Minamitake Y, Peracchia MT, Trubetskoy V, Torchilin V, Langer R. Biodegradable long-circulating polymeric nanospheres. Science. 1994;263(5153):1600-1603.

13. Gulyaev AE, Gelperina SE, Skidan IN, Antropov AS, Kivman GY, Kreuter J. Significant transport of doxorubicin into the brain with polysorbate 80-coated nanoparticles. Pharm Res. 1999;16(10): 1564-1569.

14. Wilson B, Samanta MK, Santhi K, Kumar KP, Paramakrishnan N, Suresh B. Targeted delivery of tacrine into the brain with polysorbate 80-coated poly(n-butylcyanoacrylate) nanoparticles. Eur J Pharm Biopharm. 2008;70(1):75-84.

15. Huang CY, Chen CM, Lee YD. Synthesis of high loading and encapsulation efficient paclitaxel-loaded poly(n-butyl cyanoacrylate) nanoparticles via miniemulsion. Int J Pharm. 2007;338(1-2):267-275.

16. Alyautdin RN, Petrov VE, Langer K, Berthold A, Kharkevich DA, Kreuter J. Delivery of loperamide across the blood-brain barrier with polysorbate 80 -coated polybutylcyanoacrylate nanoparticles. Pharm Res. 1997;14(3):325-328.

17. Kreuter J, Alyautdin RN, Kharkevich DA, Ivanov AA. Passage of peptides through the blood-brain barrier with colloidal polymer particles (nanoparticles). Brain Res. 1995;674(1):171-174.

18. Friese A, Seiller E, Quack G, Lorenz B, Kreuter J. Increase of the duration of the anticonvulsive activity of a novel NMDA receptor antagonist using poly(butylcyanoacrylate) nanoparticles as a parenteral controlled release system. Eur J Pharm Biopharm. 2000;49(2): 103-109.

19. Page-Clisson ME, Pinto-Alphandary H, Ourevitch M, Andremont A, Couvreur P. Development of ciprofloxacin-loaded nanoparticles: Physicochemical study of the drug carrier. J Control Release. 1998; 56(1-3):23-32.

20. Miyazaki S, Takahashi A, Kubo W, Bachynsky J, Loebenberg R. Poly n-butylcyanoacrylate (PNBCA) nanocapsules as a carrier for NSAIDs: In vitro release and in vivo skin penetration. J Pharm Pharm Sci. 2003; 6(2):238-245.

21. Michaelis K, Hoffmann MM, Dreis S, et al. Covalent linkage of apolipoprotein e to albumin nanoparticles strongly enhances drug transport into the brain. J Pharmacol Exp Ther. 2006;317(3): 1246-1253.

22. Kreuter J, Hekmatara T, Dreis S, Vogel T, Gelperina S, Langer K. Covalent attachment of apolipoprotein A-I and apolipoprotein B-100 to albumin nanoparticles enables drug transport into the brain. J Control Release. 2007;118(1):54-58.

23. Newlands ES, Stevens MF, Wedge SR, Wheelhouse RT, Brock C. Temozolomide: A review of its discovery, chemical properties, preclinical development and clinical trials. Cancer Treat Rev. 1997;23(1): 35-61.

24. Sampson JH, Archer GE, Villavicencio AT, et al. Treatment of neoplastic meningitis with intrathecal temozolomide. Clin Cancer Res. 1999;5(5):1183-1188.

25. Trinh VA, Patel SP, Hwu WJ. The safety of temozolomide in the treatment of malignancies. Expert Opin Drug Saf. 2009;8(4):493-499.

26. Reyderman L, Statkevich P, Thonoor CM, Patrick J, Batra VK, Wirth M. Disposition and pharmacokinetics of temozolomide in rat. Xenobiotica. 2004;34(5):487-500.

27. Huang G, Zhang N, Bi X, Dou M. Solid lipid nanoparticles of temozolomide: potential reduction of cardial and nephric toxicity. Int J Pharm. 2008;355(1-2):314-320. 
28. Wilson B, Samanta MK, Santhi K, Kumar KP, Paramakrishnan N, Suresh B. Poly(n-butylcyanoacrylate) nanoparticles coated with polysorbate 80 for the targeted delivery of rivastigmine into the brain to treat Alzheimer's disease. Brain Res. 2008;1200:159-168.

29. Ambruosi A, Yamamoto H, Kreuter J. Body distribution of polysorbate- 80 and doxorubicin-loaded [14C]poly(butyl cyanoacrylate) nanoparticles after i.v. administration in rats. J Drug Target. 2005;13(10):535-542.

30. Lobenberg R, Araujo L, von Briesen H, Rodgers E, Kreuter J. Body distribution of azidothymidine bound to hexyl-cyanoacrylate nanoparticles after i.v. injection to rats. J Control Release. 1998;50(1-3):21-30.

31. He W, Jiang X, Zhang ZR. Preparation and evaluation of polybutylcyanoacrylate nanoparticles for oral delivery of thymopentin. J Pharm Sci. 2008;97(6):2250-2259.

32. Wang CX, Huang LS, Hou LB, et al. Antitumor effects of polysorbate-80 coated gemcitabine polybutylcyanoacrylate nanoparticles in vitro and its pharmacodynamics in vivo on C6 glioma cells of a brain tumor model. Brain Res. 2009;1261:91-99.

33. Fawaz F, Guyot M, Lagueny AM, Devissaguet JP. Ciprofloxacinloaded polyisobutylcyanoacrylate nanoparticles: Preparation and characterization. Int J Pharm. 1997;154(2):191-203.
34. Behan N, Birkinshaw C, Clarke N. Poly n-butyl cyanoacrylate nanoparticles: A mechanistic study of polymerisation and particle formation. Biomaterials. 2001;22(11):1335-1344.

35. Muller RH, Jacobs C, Kayser O. Nanosuspensions as particulate drug formulations in therapy: Rationale for development and what we can expect for the future. Adv Drug Deliv Rev. 2001;47(1):3-19.

36. Gao K, Jiang X. Influence of particle size on transport of methotrexate across blood brain barrier by polysorbate 80 -coated polybutylcyanoacrylate nanoparticles. Int J Pharm. 2006;310(1-2):213-219.

37. Alyautdin RN, Tezikov EB, Ramge P, Kharkevich DA, Begley DJ, Kreuter J. Significant entry of tubocurarine into the brain of rats by adsorption to polysorbate 80 -coated polybutylcyanoacrylate nanoparticles: An in situ brain perfusion study. J Microencapsul. 1998;15(1): 67-74.

38. Sun WQ, Xie CS, Wang HF, Hu Y. Specific role of polysorbate 80 coating on the targeting of nanoparticles to the brain. Biomaterials. 2004;25(15):3065-3071.

39. Kreuter J. Nanoparticulate systems for brain delivery of drugs. Adv Drug Deliv Rev. 2001;47(1):65-81.
International Journal of Nanomedicine

\section{Publish your work in this journal}

The International Journal of Nanomedicine is an international, peerreviewed journal focusing on the application of nanotechnology in diagnostics, therapeutics, and drug delivery systems throughout the biomedical field. This journal is indexed on PubMed Central, MedLine, CAS, SciSearch ${ }^{\circledR}$, Current Contents ${ }^{\circledR} /$ Clinical Medicine, Journal

\section{Dovepress}

Citation Reports/Science Edition, EMBase, Scopus and the Elsevier Bibliographic databases. The manuscript management system is completely online and includes a very quick and fair peer-review system, which is all easy to use. Visit http://www.dovepress.com/ testimonials.php to read real quotes from published authors. 